\title{
Density functional theory for hard spherocylinders: phase transitions in the bulk and in the presence of external fields
}

\author{
Hartmut Graf and Hartmut Löwen $\dagger$ \\ Institut für Theoretische Physik II, Heinrich-Heine-Universität Düsseldorf, Universitätsstraße 1,
} D-40225 Düsseldorf, Germany

Received 10 November 1998

\begin{abstract}
The phase behaviour of hard spherocylinders is calculated using the classical density functional theory of freezing. In particular, we construct a modified weighted-density approximation and calculate the bulk phase diagram. While the stability regime of the isotropic, nematic and smectic-A phases is in reasonable agreement with computer simulation data, the theory fails to describe the correct stability of the crystalline phases. Furthermore, we investigate the phase behaviour in an external field coupling to the orientational degrees of freedom. As a result, bulk phases which have the same translational symmetry but a different orientational symmetry can be transformed continuously into each other above a critical strength of the external field.
\end{abstract}

\section{Introduction}

Colloidal suspensions of rod-like macromolecules are ideal model systems exhibiting liquidcrystalline phase transformations. One prominent example is an aqueous solution of tobacco mosaic viruses [2]. In a theoretical description one usually starts with the interactions between the rods, and then predicts the phase behaviour as a function of the thermodynamic parameters. Since this is a formidable task, in general, one typically tries to keep the model as simple as possible in order to reduce the relevant parameters. One of the simplest non-trivial models comprising an anisotropic interaction is that of hard spherocylinders (HSC) where solely excluded-volume forces are considered. This immediately implies that the temperature is an irrelevant variable. A spherocylinder consists of a cylinder of length $L$ and diameter $D$ capped with two hemispheres of the same diameter $D$ at the ends. The HSC model can be used to describe lyotropic liquid crystals. It has the further advantage that its isotropic limit $L \rightarrow 0$ leads to the well-known hard-sphere model.

The HSC system has attracted attention since the early calculations of Onsager [28], who demonstrated the existence of a first-order isotropic-nematic phase transformation for large aspect ratios $p=L / D$. The full phase behaviour for arbitrary length-to-width ratios was recently explored by computer simulations by Bolhuis and Frenkel [3]. The phase diagram obtained was very rich including stable isotropic, nematic, smectic-A, plastic crystalline and fully crystalline phases with both $\mathrm{ABC}$ and AAA stacking sequences. Subsequently we proposed a simple theory $[9,10]$ combining scaled-particle methods for the disordered phases with a cell description for the solids. This cell theory was able to reproduce the overall phase behaviour with two empirical fitting parameters. In this paper we shall use a density functional approach to predict the phase diagram. The reason for doing this is twofold: first, a complete ' $a b$ initio' description is wanted avoiding any heuristic ingredients; second, once a density

$\dagger$ Also at: Institut für Festkörperforschung, Forschungszentrum Jülich, D-52425 Jülich, Germany. 
functional is found describing the bulk phases, in principle it can be directly used to calculate the influence of external fields on these phase transitions.

For hard spheres, different approximations for the density functionals are available which give in general excellent results for the freezing transition; for a review see e.g. [8, 22, 29]. A more complicated but less-studied system is that of strictly aligned non-spherical hard particles avoiding the complexity arising from the statistical average of the orientational degrees of freedom. For parallel hard spherocylinders, the smectic branch was determined by searching for instabilities in the nematic phase $[14,26,45]$. Also the solid phase was included in such models [57].

A full consideration of the additional orientational degrees of freedom complicates the calculations enormously. Besides the Onsager approach for infinitely long rods only few attempts have been made to compute the phase behaviour of finite-length particles using scaled-particle [18] and density functional theory (DFT). As regards the latter, there is the approximation of Parsons where the functional is appropriately scaled to map onto the excess free energy of the spherical fluid phase [30]. This theory reproduces the isotropic-nematic transition fairly well [20]. The approach has also been used to include the smectic phase via a stability analysis of the nematic phase $[6,7,46,47]$. Furthermore, a weighted-density approach was put forward by Poniewierski and Holyst [33,34].

The aim of our paper is twofold: first, we make an attempt to describe the full phase diagram of HSC including all crystalline phases by density functional theory; second, we study phase transformations in the presence of an external field. In the density functional approach, we have used the weighted-density approximation $[33,34]$ together with a modification used for hard spheres by Denton and Ashcroft [5]. As a result, however, this version of DFT is not capable of reproducing the phase diagram completely. In particular, the smectic phase always 'beats' the fully crystalline phase even at high packing fractions, which is clearly an artifact of the theory. On the other hand, the less-ordered phases are well described and all of their phase coexistences are in good overall agreement with the computer simulation data.

Once a density functional description is established for the bulk phases, it can be used to study phase transitions in an external field. In this paper we study an external field which couples to the orientational degrees of freedom and investigate how the different liquidcrystalline transitions are affected by the field. This question has been studied previously only for the isotropic-nematic transition, both theoretically $[13,15,17,52,56]$ and experimentally $[21,27,49]$. In particular, it has been seen that the isotropic-nematic transition vanishes above a critical field strength. In the present work we extend the analysis to other transitions, including the smectic-A, the plastic and the fully crystalline phases. As theoretical tools, we use both density functional theory and the cell model of references $[9,10]$. Our main result is that any two phases which possess the same translational symmetry can be transformed continuously into each other if a critical threshold of the field strength is reached.

The paper is organized as follows. In section 2 we develop the modified weighted-density functional theory for HSC and discuss the resulting phase behaviour. An external field acting only on the orientational degrees of freedom is introduced in section 3. We finish with a forward look in section 4.

\section{Modified weighted-density functional theory for hard spherocylinders}

\subsection{The model and basics of density functional theory}

We consider a system of $N$ hard spherocylinders in a volume $V$ with a finite number density $\rho=N / V$. The cylindrical part of the rods has a length $L$ and a width $D$; hence the aspect 
ratio is $p=L / D$. It is convenient to scale densities in terms of the close-packed density

$$
\rho_{\mathrm{CP}}=\frac{2}{(\sqrt{2}+\sqrt{3} p) D^{3}}
$$

i.e. we use a reduced density $\rho^{*}=\rho / \rho_{\mathrm{CP}}$. The phase diagram is completely governed by these two parameters, $p$ and $\rho^{*}$.

The key quantity of density functional theory is the one-particle density $\rho^{(1)}(\vec{r}, \vec{\omega})$ depending both on the translational degree of freedom, $\vec{r}$, and the orientational degree of freedom, $\vec{\omega}$. Here, $\vec{r}$ is an arbitrary three-dimensional vector while $\vec{\omega}$ is a three-dimensional unit vector.

In the following we present the essential features of the modified weighted-density approximation (MWDA). We start by writing the density functional of the Helmholtz free energy as

$$
\mathcal{F}\left[\rho^{(1)}\right]=\mathcal{F}^{\mathrm{id}}\left[\rho^{(1)}\right]+\mathcal{F}^{\mathrm{ex}}\left[\rho^{(1)}\right]
$$

where the ideal-gas part

$$
\mathcal{F}^{\mathrm{id}}\left[\rho^{(1)}\right]=k_{\mathrm{B}} T \int \mathrm{d} \boldsymbol{x} \rho^{(1)}(\boldsymbol{x})\left[\ln \left(\Lambda^{3} \rho^{(1)}(\boldsymbol{x})\right)-1\right]
$$

is known exactly. $k_{\mathrm{B}} T$ is the thermal energy and $\Lambda$ the (spatial) thermal wavelength, and we introduced the notation $\boldsymbol{x}=(\vec{r}, \vec{\omega})$ for the configuration variables. The obvious meaning of $\int \mathrm{d} x \cdots$ is $\int_{V} \mathrm{~d}^{3} \vec{r} \int_{S_{2}} \mathrm{~d}^{2} \vec{\omega} \cdots$ where the integration runs over the unit sphere where

$$
\int_{S_{2}} d^{2} \vec{\omega}=\int_{0}^{2 \pi} \mathrm{d} \phi \int_{0}^{\pi} \sin (\theta) \mathrm{d} \theta=4 \pi .
$$

Different density functional theories approximate the excess part on different levels. Let us first write

$$
\mathcal{F}^{\mathrm{ex}}\left[\rho^{(1)}\right]=\int \mathrm{d} \boldsymbol{x} \rho^{(1)}(\boldsymbol{x}) \Psi^{\mathrm{inh}}\left(\left[\rho^{(1)}\right] ; \boldsymbol{x}\right)
$$

where the excess free energy per particle of the inhomogeneous fluid at the configuration point $\boldsymbol{x}$ is denoted by $\Psi^{\mathrm{inh}}\left(\left[\rho^{(1)}\right] ; \boldsymbol{x}\right)$.

In the weighted-density approximation $[4,50]$, the functional $\Psi^{\text {inh }}$ is replaced by the corresponding expression of the uniform system, evaluated however at a reference density $\bar{\rho}$ :

$$
\Psi^{\mathrm{inh}}\left(\left[\rho^{(1)}\right] ; \boldsymbol{x}\right) \equiv \Psi[\bar{\rho}(\boldsymbol{x})]
$$

In this approximation some correlations are taken into account non-perturbatively. $\bar{\rho}$ is a weighted average of the actual density

$$
\bar{\rho}\left(\boldsymbol{x}_{1}\right)=\int \mathrm{d} \boldsymbol{x}_{2} w\left(\boldsymbol{x}_{1}, \boldsymbol{x}_{2}\right) \rho^{(1)}\left(\boldsymbol{x}_{2}\right)
$$

where the weight function $w\left(\boldsymbol{x}_{1}, \boldsymbol{x}_{2}\right)$ has to fulfil the normalization constraint $\int \mathrm{d} \boldsymbol{x} w(\boldsymbol{x})=1$ and typically depends on the weighted density $\bar{\rho}$ itself.

The modified weighted-density approximation [5] proceeds one step further by averaging the weighted density with the actual density

$$
\hat{\rho}=\frac{1}{N} \int \mathrm{d} \boldsymbol{x}_{1} \rho^{(1)}\left(\boldsymbol{x}_{1}\right) \bar{\rho}\left(\boldsymbol{x}_{1}\right) .
$$

Consequently the excess free energy per particle becomes now a global quantity

$$
\frac{\mathcal{F}^{\mathrm{ex}}}{N}=\Psi(\hat{\rho})
$$

independent of the configuration point $\boldsymbol{x}$, and the weight function turns out to be proportional to the direct correlation function [5]. 


\subsection{The approximations of the MWDA}

To apply the modified weighted-density approach one has to choose several ingredients: the form of the bulk excess free energy for a uniform system, the weight function and the actual parametrization of the density which we describe now in more detail.

2.2.1. Approximation of the excess free energy. For hard spheres, the excess free energy of a homogeneous system can be obtained analytically within the Percus-Yevick approximation or equivalently by using a scaled-particle theory. Higher virial coefficients are incorporated heuristically by the Carnahan-Starling expression [12], where the reduced excess free energy per particle reads

$$
\beta \Psi_{\mathrm{CS}}=\eta(4-3 \eta) /(1-\eta)^{2} .
$$

Here $\eta=\rho v_{0}$ denotes the packing fraction, $v_{0}$ is the volume of a single particle (sphere or spherocylinder) and $\beta \equiv 1 / k_{B} T$. Much less is known for hard spherocylinders. It is only the second virial coefficient $B_{2}=\pi\left(2 D^{3} / 3+L D^{2}+L^{2} D / 4\right)$ which is known analytically [28]. Still an approximative scaled-particle theory can be performed [1].

In this study, we choose a different approach following Holyst and co-workers [33,34]: the excess free energy $\Psi$ is approximated by a virial expansion, where one takes the exactly known second virial coefficient $B_{2}$ for hard spherocylinders and approximates all higher virial coefficients with the corresponding ones of hard spheres at the same packing fraction $\eta$, namely by the Carnahan-Starling formula for $\Psi_{\mathrm{CS}}$ :

$$
\beta \Psi(\eta)=\rho B_{2}+\beta \Psi_{\mathrm{CS}}-4 \eta .
$$

The equation of state from this functional yields better agreement than the one from scaledparticle theory [1] when compared to simulation data [3]. Also notable is that this formula interpolates between the two limiting cases, hard spheres $(p=0)$ and infinitely long rods $(p \rightarrow \infty)$ at low densities.

2.2.2. Approximation for the weight function. As noted above, the weight function of hard spheres in the MWDA is proportional to the direct correlation function. As the direct correlation function for hard spherocylinders is unknown, we take the low-density approximation, namely the normalized Mayer function $f_{2}$ :

$$
w\left(\vec{r}_{2}-\vec{r}_{1}, \vec{\omega}_{1}, \vec{\omega}_{2}\right)=-\frac{f_{2}\left(\vec{r}_{2}-\vec{r}_{1}, \vec{\omega}_{1}, \vec{\omega}_{2}\right)}{2 B_{2}} .
$$

The weight function is thus a step function, measuring whether the particles sited at the space points $\vec{r}_{1}$ and $\vec{r}_{2}$ and pointing in the directions $\vec{\omega}_{1}$ and $\vec{\omega}_{2}$ overlap or not:

$$
w\left(\vec{r}_{2}-\vec{r}_{1}, \vec{\omega}_{1}, \vec{\omega}_{2}\right)= \begin{cases}1 /\left(2 B_{2}\right) & \text { for overlap } \\ 0 & \text { otherwise. }\end{cases}
$$

We remark that in one of the original versions of the weighted-density theory, Tarazona [50] applied such a weight function successfully to the freezing transition of hard spheres.

2.2.3. Parametrization of the density. The density functional has to be minimized with respect to the one-particle density $\rho^{(1)}(\vec{r}, \vec{\omega})$ at fixed temperature and fixed average density. For $\rho^{(1)}(\vec{r}, \vec{\omega})$ we use a factorization ansatz assuming that the orientational and translational degrees of freedom are decoupled:

$$
\rho^{(1)}(\vec{r}, \vec{\omega})=\rho(\vec{r}) g(\vec{\omega})
$$


where $g(\vec{\omega})$ is the orientation distribution function and $\rho(\vec{r})$ the angular averaged density. Obviously, the function $g(\vec{\omega})$ can be obtained by integrating over the translational degrees of freedom: $g(\vec{\omega})=N^{-1} \int \mathrm{d}^{3} \vec{r} \rho^{(1)}(\vec{r}, \vec{\omega})$ and should satisfy the normalization condition $\int_{S_{2}} \mathrm{~d}^{2} \vec{\omega} g(\vec{\omega})=1$. Henceforth we use the explicit parametrization introduced by Onsager [28]:

$$
g(\vec{\omega})=\frac{\alpha}{4 \pi \sinh \alpha} \cosh \left(\alpha \vec{\omega} \cdot \vec{\omega}_{0}\right)
$$

where $\alpha$ is the single variational parameter describing the width of the orientation distribution around a given director $\vec{\omega}_{0}$. The solid structure contained in $\rho(\vec{r})$ will be parametrized differently in the various phases as described later.

\subsection{Description of the different phases}

2.3.1. The isotropic phase. The one-particle density in the isotropic phase is homogeneous:

$$
\rho_{\text {iso }}^{(1)}(\vec{r}, \vec{\omega})=\rho /(4 \pi)
$$

with the overall number density $\rho$. As the density functional theory starts from the uniformfluid phase, the modified weighted density (7) and the overall density $\rho$ coincide: $\hat{\rho} \equiv \hat{\rho}_{\text {iso }}=\rho$. The ideal-gas part (3) of the free energy per particle reads

$$
\frac{\beta \mathcal{F}^{\text {id }}}{N}=\ln \left(\frac{\Lambda^{3}}{4 \pi} \rho\right)-1
$$

The excess free energy per particle is given by (4) and (9) as

$$
\frac{\beta \mathcal{F}_{\text {iso }}^{\text {ex }}}{N}=\frac{2 / 3+p+p^{2} / 4}{1 / 6+p / 4} \eta+\frac{5 \eta^{2}-4 \eta^{3}}{(1-\eta)^{2}} .
$$

where $\eta=\rho v_{0}$ is the packing fraction.

2.3.2. The nematic phase. In the nematic phase, the particles point along a preferred direction described by the orientation distribution $g(\vec{\omega})(13)$, while their centres of mass are still homogeneously distributed. Therefore the density parametrization now reads

$$
\rho_{\text {nem }}^{(1)}(\vec{r}, \vec{\omega})=\rho g(\vec{\omega}) .
$$

The ideal-gas term (3) now contains besides the part describing the translational degrees of freedom (15) another part corresponding to ideal rotators obeying the orientation distribution $g(\vec{\omega}):$

$$
\frac{\beta \mathcal{F}^{\text {id,rot }}}{N}=\int \mathrm{d}^{2} \vec{\omega} g(\vec{\omega}) \ln [4 \pi g(\vec{\omega})] .
$$

According to the above prescription, the excess free energy has to be evaluated at the weighted density (7):

$\hat{\rho}_{\text {nem }}=\frac{1}{N} \int \mathrm{d}^{3} \vec{r}_{1} \mathrm{~d}^{2} \vec{\omega}_{1} \rho_{\text {nem }}^{(1)}\left(\vec{r}_{1}, \vec{\omega}_{1}\right) \int \mathrm{d}^{3} \vec{r}_{2} \mathrm{~d}^{2} \vec{\omega}_{2} w\left(\vec{r}_{2}-\vec{r}_{1}, \vec{\omega}_{1}, \vec{\omega}_{2}\right) \rho_{\text {nem }}^{(1)}\left(\vec{r}_{2}, \vec{\omega}_{2}\right)$

which can be calculated analytically [28]:

$\hat{\rho}_{\text {nem }}(\alpha)=\rho \frac{2 / 3+p+p^{2} I_{2}(2 \alpha) /\left(2 \sinh ^{2}(\alpha)\right)}{2 / 3+p+(1 / 4) p^{2}} \equiv \rho \frac{\left\langle\left\langle v_{\mathrm{excl}}\left(\vec{\omega}_{1}, \vec{\omega}_{2}\right)\right\rangle_{g\left(\vec{\omega}_{1}\right)}\right\rangle_{g\left(\vec{\omega}_{2}\right)}}{2 B_{2}}$

where $I_{2}(x)$ is a spherical Bessel function of real argument $x$. Note that due to our choice of the weight function, the weighted density is proportional to the double angle-averaged excluded volume $\left.\left\langle\left\langle v_{\text {excl }}\left(\vec{\omega}_{1}, \vec{\omega}_{2}\right)\right\rangle_{g\left(\vec{\omega}_{1}\right)}\right)\right\rangle_{g\left(\vec{\omega}_{2}\right)}$ of two spherocylinders pointing in directions $\vec{\omega}_{1}$ 
and $\vec{\omega}_{2}$. Here, the brackets $\langle\cdots\rangle_{g(\vec{\omega})}$ denote the angular average with respect to the orientation distribution $g(\vec{\omega})$ :

$$
\langle\cdots\rangle_{g(\vec{\omega})}=\int_{S_{2}} \mathrm{~d}^{2} \vec{\omega} g(\vec{\omega}) \cdots
$$

To summarize, the free energy in the nematic phase reads

$$
\mathcal{F}_{\text {nem }}=\mathcal{F}^{\text {id }}+\mathcal{F}^{\text {id,rot }}+N \Psi\left(\hat{\eta}_{\text {nem }}(\alpha)\right)
$$

with $\hat{\eta}_{\text {nem }}=\hat{\rho}_{\text {nem }} v_{0}$. Finally, a minimization of $\mathcal{F}_{\text {nem }}$ has to be performed with respect to $\alpha$ which is done numerically [36].

2.3.3. The smectic-A phase. In the smectic-A phase, the particles are arranged in layers perpendicular to the director. We model this by a modulation of the density along the director, i.e. parametrize the density as

$$
\rho_{\mathrm{sm}}^{(1)}(\vec{r}, \vec{\omega})=\rho \Xi(k z) g(\vec{\omega})
$$

where the director points along the $z$-axis for convenience, $\vec{r}=(x, y, z)$ and $k=2 \pi / d$ is the smectic wavelength related to the layer spacing $d$. As a trial function for the modulation of the density we use

$$
\Xi(k z)=\frac{1}{N_{\Xi}} \exp \left[\sum_{n=1}^{4} \xi_{n} \cos (n \cdot k z)\right]
$$

where we include up to four variational parameters $\xi_{n}, n=1,2,3,4$. $N_{\Xi}$ is present to guarantee the correct normalization, $\int_{0}^{2 \pi} \Xi(\lambda) \mathrm{d} \lambda=1$.

An additional contribution to the ideal-gas free energy arises from the confinement of the particles in layers. The ideal free energy (3) thus reads

$$
\frac{\beta \mathcal{F}_{\mathrm{sm}}^{\mathrm{id}}}{N}=\ln \left(\frac{\Lambda^{3}}{4 \pi} \rho\right)-1+\int_{S_{2}} \mathrm{~d}^{2} \vec{\omega} g(\vec{\omega}) \ln [4 \pi g(\vec{\omega})]+\frac{1}{2 \pi} \int_{0}^{2 \pi} \Xi(\lambda) \ln [\Xi(\lambda)] \mathrm{d} \lambda .
$$

The main effort when making calculations for the smectic phase is in the evaluation of the weighted density. To overcome the problem of calculating the high-dimensional integral in equation (7), we make use of an expansion in spherical harmonics (see the appendix for details). We expand the weight function as

$$
w\left(\vec{r}_{12}, \vec{\omega}_{1}, \vec{\omega}_{2}\right)=\sum_{\Lambda} \hat{w}_{\Lambda}\left(r_{12}\right) \Phi_{\Lambda}\left(\vec{\omega}_{12}, \vec{\omega}_{1}, \vec{\omega}_{2}\right)
$$

where $\Phi_{\Lambda}\left(\vec{\omega}_{12}, \vec{\omega}_{1}, \vec{\omega}_{2}\right)$ are the spherical invariants (A.1) and the summation runs over the triple

$$
\Lambda=\left(l_{1}, l_{2}, l\right) \in \mathbb{N}_{0}^{3} .
$$

The expansion coefficients can be obtained from (A.4)

$$
\begin{array}{r}
\hat{w}_{\Lambda}\left(r_{12}\right)=\sqrt{\frac{4 \pi}{2 l+1}} \int \mathrm{d}^{2} \vec{\omega}_{1} \int \mathrm{d}^{2} \vec{\omega}_{2} w\left(r_{12}, \vec{\omega}_{1}, \vec{\omega}_{2} ; \vec{\omega}_{12}=\hat{e}_{z}\right) \\
\times \sum_{m=-\min \left(l_{1}, l_{2}\right)}^{m=\min \left(l_{1}, l_{2}\right)} C\left(l_{1} l_{2} l, m \bar{m} 0\right) \mathcal{Y}_{l_{1} \bar{m}}\left(\vec{\omega}_{1}\right) \mathcal{Y}_{l_{2} m}\left(\vec{\omega}_{2}\right) .
\end{array}
$$

The spherical harmonics are denoted by $\mathcal{Y}_{l m}$, and $\bar{m}=-m$, as introduced in the appendix.

When now combining equations (7), (23) and (28) one can make extensive use of symmetry properties of the spherical harmonics [11] and finally obtain

$\hat{\rho}_{\mathrm{sm}}=\frac{1}{N} \int \mathrm{d}^{3} \vec{r} \rho(\vec{r}) \bar{\rho}(\vec{r})$ 
with

$$
\begin{aligned}
\bar{\rho}(\vec{r})=\rho \sum_{\Lambda} \int & \mathrm{d}^{3} \vec{r}_{12} \hat{w}_{\Lambda}\left(r_{12}\right) \mathcal{Y}_{l 0}\left(\vec{\omega}_{12}\right) \Phi\left(k z-k z_{12}\right) \\
& \times C\left(l_{1} l_{2} l, 000\right) \int \mathcal{Y}_{l_{1} 0}\left(\vec{\omega}_{1}\right) g\left(\vec{\omega}_{1}\right) \mathrm{d}^{2} \vec{\omega}_{1} \int \mathcal{Y}_{l_{2} 0}\left(\vec{\omega}_{2}\right) g\left(\vec{\omega}_{2}\right) \mathrm{d}^{2} \vec{\omega}_{2} .
\end{aligned}
$$

The total free energy in the smectic phase

$$
\mathcal{F}_{\text {sm }}=\mathcal{F}_{\text {sm }}^{\text {id }}+N \Psi\left(\hat{\eta}_{\text {sm }}\right)
$$

is finally numerically minimized with respect to $\alpha$, the smectic order parameters $\left\{\xi_{n}\right\}$ and the layer spacing $d$.

2.3.4. Crystalline phases. We restrict our attention to two different crystalline structures, namely the plastic solid and the ABC crystal, whereas other candidate structures such as an AAA crystal as well as a bcc solid will be excluded from consideration here due to the tremendous numerical effort that they would engender. We first introduce basic features and afterwards specialize to the two different phases.

( $\alpha$ ) General ingredients. A sufficient density parametrization for the hard-sphere crystal are Gaussian peaks centred at the lattice positions. For HSC the widths of the Gaussians parallel and perpendicular to the director might be different, but as a first approximation we choose an isotropic peak. For further computational convenience we fix the ratio of distortion, i.e. the ratio of the two different lattice constants.

The translational part of the density is expressed in terms of Fourier coefficients $\rho_{\vec{G}}$

$$
\rho_{\text {sol }}(\vec{r})=\rho\left(1+\sum_{\vec{G}}^{\prime} \rho_{\vec{G}} \mathrm{e}^{\mathrm{i} \vec{G} \cdot \vec{r}}\right)
$$

where the summation runs over all non-zero reciprocal-lattice vectors $\vec{G}$. The Fourier coefficients read $\rho_{\vec{G}}=\exp \left[-\vec{G}^{2} / 4 \gamma\right]$, where $\gamma$ describes the width of the Gaussians in real space. Inserting equation (32) into the expression for the weighted density (6) we get

$$
\begin{aligned}
\bar{\rho}_{\text {sol }}(\vec{r})=\rho\left\langle\left\langle\frac{v_{\text {excl }}\left(\vec{\omega}_{1}, \vec{\omega}_{2}\right)}{2 B_{2}}\right\rangle_{g\left(\vec{\omega}_{1}\right)}\right\rangle_{g\left(\vec{\omega}_{2}\right)} & \\
& +\rho \sum_{\vec{G}}^{\prime} \rho_{\vec{G}} \mathrm{e}^{\mathrm{i} \vec{G} \cdot \vec{r}}\left\langle\left\langle\int \mathrm{d}^{3} \vec{r}^{\prime} w\left(\vec{r}^{\prime}, \vec{\omega}_{1}, \vec{\omega}_{2}\right) \mathrm{e}^{\mathrm{i} \vec{G} \cdot \vec{r}^{\prime}}\right\rangle_{g\left(\vec{\omega}_{1}\right)}\right\rangle_{g\left(\vec{\omega}_{2}\right)}
\end{aligned}
$$

and finally

$\hat{\rho}_{\mathrm{sol}}=\rho\left\langle\left\langle\frac{v_{\mathrm{excl}}\left(\vec{\omega}_{1}, \vec{\omega}_{2}\right)}{2 B_{2}}\right\rangle_{g\left(\vec{\omega}_{1}\right)}\right\rangle_{g\left(\vec{\omega}_{2}\right)}+\rho \sum_{\vec{G}}^{\prime} \rho_{\vec{G}}^{2}\left\langle\left\langle\tilde{w}\left(\vec{G}, \vec{\omega}_{1}, \vec{\omega}_{2}\right)\right\rangle_{g\left(\vec{\omega}_{1}\right)}\right\rangle_{g\left(\vec{\omega}_{2}\right)}$.

Here, the Fourier transform of the weight function with respect to the spatial coordinate is denoted by $\tilde{w}\left(\vec{G}, \vec{\omega}_{1}, \vec{\omega}_{2}\right)$.

For an evaluation of the modified weighted density one can make use of an expansion of the weight function (26), rearranging some terms, as

$$
w\left(\overrightarrow{r_{12}}, \vec{\omega}_{1}, \vec{\omega}_{2}\right) \equiv \sum_{\Lambda, \Gamma} w_{\Lambda, \Gamma}^{I}\left(\vec{r}_{12}\right) w_{\Lambda, \Gamma}^{I I}\left(\vec{\omega}_{1}, \vec{\omega}_{2}\right) .
$$

The summation runs now additionally over the triple

$$
\Gamma=\left(m_{1}, m_{2}, m\right) .
$$


Plugging this into equation (34), the modified weighted density $\hat{\rho}$ is most easily expressed in terms of the Fourier-transformed coefficients $\tilde{w}_{\Lambda, \Gamma}^{I}$ of the expansion of the weight function (35):

$\hat{\rho}_{\mathrm{sol}}=\rho\left\langle\left\langle\frac{v_{\mathrm{excl}}\left(\vec{\omega}_{1}, \vec{\omega}_{2}\right)}{2 B_{2}}\right\rangle_{g\left(\vec{\omega}_{1}\right)}\right\rangle_{g\left(\vec{\omega}_{2}\right)}+\rho \sum_{\vec{G}}^{\prime} \rho_{\vec{G}}^{2} \sum_{\Lambda} \tilde{w}_{\Lambda, 0}^{I}(\vec{G})\left\langle\left\langle w_{\Lambda, 0}^{I I}\left(\vec{\omega}_{1}, \vec{\omega}_{2}\right)\right\rangle_{g\left(\vec{\omega}_{1}\right)}\right\rangle_{g\left(\vec{\omega}_{2}\right)}$.

This reduces the summation over $\Gamma$ to the contribution $\Gamma=(0,0,0)$. Evaluation of this final expression for the modified weighted density is still tedious. As we perform the expansion of the weight function around the isotropic state, the calculations require increasing effort for an increasing length-to-width ratio of the particles. However, especially for the orientationally isotropic case, this expression is advantageous.

( $\beta$ ) Plastic crystal. In a plastic crystal the particles are located on fcc-lattice positions and pointing in arbitrary directions. The lattice constant is thus fixed by the density. In this phase, symmetry properties of the spherical harmonics reduce the summation in the weighted density (36) to just the contribution $\Lambda=(0,0,0)$. The minimization is finally performed only with respect to the width $\gamma$ of the density distribution.

( $\gamma$ ) ABC crystal. In the orientational ordered solid the slowly converging expansion of the weight function forces us to approximate the density further: we treat only the zeroth density mode with the full orientational dependence and approximate all higher density modes by the corresponding ones for strictly aligned particles, i.e.

$$
\rho_{\text {sol }}(\vec{r}, \vec{\omega})=\rho g(\vec{\omega})+\rho \sum_{\vec{G}}^{\prime} \rho_{\vec{G}} \mathrm{e}^{\mathrm{i} \overrightarrow{\vec{G}} \cdot \vec{r}} \delta(\vec{\omega}) .
$$

On this assumption, the double angular average for the non-zero density modes in the modified weighted density (34) has only to be evaluated for strictly aligned particles. Thus, the modified weighted density finally reads

$$
\hat{\rho}_{\text {sol }}=\rho\left\langle\left\langle\frac{v_{\text {excl }}\left(\vec{\omega}_{1}, \vec{\omega}_{2}\right)}{2 B_{2}}\right\rangle_{g\left(\overrightarrow{\omega_{1}}\right)}\right\rangle_{g\left(\overrightarrow{\omega_{2}}\right)}+\rho \sum_{\vec{G}}^{\prime} \rho_{\vec{G}}^{2} \tilde{w}_{\|}(\vec{G})
$$

with $\tilde{w}_{\|}(\vec{G})$ being the Fourier transform of the weight function for strictly aligned particles.

The lattice of the ABC crystal is stretched in the direction of the director by a factor $p+1$, as inspired by the close-packed configuration. The final minimization has to be performed with respect to the orientational as well as the translational order.

\subsection{Results for the phase diagram and discussion}

Density functional results for the phase diagram as a function of $p$ and the reduced density $\rho^{*}$ are given in figure 1. The vertical grey bar separates two distinct regions. First, for $p<3$, the isotropic fluid (I) freezes into a crystalline structure, either a plastic crystal (P) or an ABC-stacked solid. The agreement with the computer simulation data [3] shown as dots in figure 1 is reasonable. However, the result is spoiled by the fact that the smectic free energy is always lower than the free energy of the orientational ordered crystal. Consequently we have disregarded the smectic phase in this part of the phase diagram. For $p>3$, on the other hand, the full cascade of liquid-crystalline phases is obtained, namely the fluid, nematic $(\mathrm{N})$ and smectic-A (SmA) phases. Again, the agreement with the computer simulation data is fair, but, within our description, the solid phase is only metastable. 


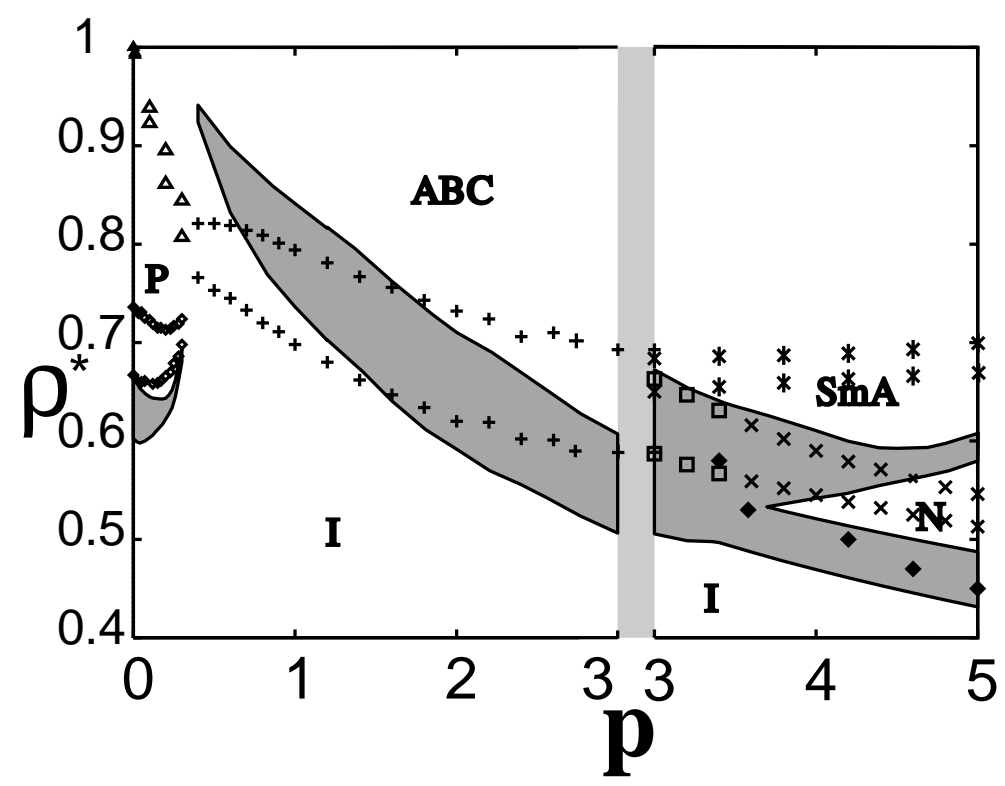

Figure 1. The phase diagram of hard spherocylinders obtained by density functional theory in the $\rho^{*}-p$ plane: the coexistence regions are shown as shaded areas and we include simulational [3] results as dots. There is an aligned $\mathrm{ABC}$ solid, a plastic crystal (P) and an isotropic fluid (I) in the left-hand part of the diagram, where the smectic phase has to be neglected. To the right from the vertical grey bar we observe besides the fluid a nematic $(\mathrm{N})$ and a smectic-A $(\mathrm{SmA})$ phase. The meanings of the symbols for the simulational data are: + : I-ABC transition; $\diamond: \mathrm{I}-\mathrm{P}$ transition; $\square: \mathrm{I}-\mathrm{SmA}$ transition; $\mathrm{I}-\mathrm{N}$ transition; $\times: \mathrm{N}-\mathrm{SmA}$ transition; $*: \mathrm{SmA}-\mathrm{ABC}$ transition; $\triangle: \mathrm{P}-\mathrm{ABC}$ transition.

We remark that recent numerical studies examined the isotropic fluid-plastic-crystal transition for hard spherocylinders [53], and also for other hard convex bodies expected to behave similarly to short HSC, such as hard dumb-bells [41,54]. Within density functional calculations, coexistence densities have been calculated for hard ellipsoids $[23,43]$ and hard dumb-bells $[25,42,44]$. Our approach here has an accuracy comparable to that of the original approach of Tarazona [50] for the freezing of hard spheres and thus underestimates the transition densities. This behaviour is continued for non-zero length-to-width ratios and we therefore underestimate the coexistence densities of the fluid-plastic transition.

In computer simulations the isotropic-nematic transition appears to be first order; however, it shows no measurable density jump. All different DFT including our MWDA [20, 33, 34] describe the transition region fairly well, but overestimate the density jump. Note that, by construction, the Onsager limit is included exactly in our description.

The slope of the nematic-smectic transition had been under discussion until it was determined by the computer simulations of Bolhuis and Frenkel [3], who demonstrated that it is negative. Poniewierski and co-workers found [33-35] an increase of the transition densities as the length of the rods increases. On the other hand, Somoza and Tarazona [47] find the opposite behaviour. As we follow the description of Poniewierski, we find the wrong increasing behaviour as well, however, with a small slope. The debate about the nature of the nematicsmectic transition is another point: for parallel hard spherocylinders it was claimed that the $\mathrm{N}-$ SmA transition shows a Kosterlitz-Thouless-like, continuous behaviour. All different density functional theories predicted a tricritical point for rods with an orientational degree of freedom. 
Poniewierski [32] has shown that the transition is continuous in the Onsager limit if the Onsager approach (including only the second virial coefficient) is used, but is first order if the third virial coefficient is taken into account. Recent computer simulations by Polson and Frenkel [31] have clarified the situation: even in the Onsager limit the nematic-smectic transition remains first order.

The striking fact of our MWDA theory is that the free energy of the smectic phase is lower than that of the $\mathrm{ABC}$ crystal. This might be due to the restricted density parametrization in the $\mathrm{ABC}$ crystalline phases. It is more probable, however, that it is an artifact due to the fact that the smectic structure in our description does not satisfy the close-packed limit within layers. In order to remedy this deficiency one should start with different functionals which possess the correct limit of close packing. The geometrical-measure-based functional of Rosenfeld [38-40] which is in principle applicable to orientable convex bodies fulfils this requirement.

\section{Orientation-dependent external fields}

\subsection{The model}

Anisotropic particles can be oriented by an external electric or magnetic field. For instance, the tobacco mosaic virus has a positive anisotropy of the diamagnetic susceptibility and is thus aligned along an external magnetic field. We model an external aligning field by the external potential

$$
U_{\text {ext }}(\vec{\omega})=-U_{0} \cos ^{2}\left(\vec{\omega} \cdot \vec{\omega}_{0}\right)
$$

acting on each rod where $\vec{\omega}_{0}$ is a unit vector in the direction of the external field and $U_{0}$ is the strength of the external interaction. The angle between $\vec{\omega}$ and $\vec{\omega}_{0}$ is denoted by $\theta$ in the following. This type of interaction which is certainly the dominating term in any orientational coupling has been considered previously to model rod-like polyelectrolytes in an external electric field [24]. We remark that the limit $U_{0} \rightarrow \infty$ reduces the model to that of strictly aligned rods. Hence the orientational degrees of freedom can be continuously frozen in as the field is increasing.

The basic question is that of how the external field shifts and influences the phase boundaries of the bulk phase diagram. Theoretical calculations were first done by Khoklov and Semenov [17]: within the Onsager theory (i.e. assuming the particles to be infinitely long and using the Onsager trial function (13)), but also for semi-flexible particles, they estimated the influence of various types of external field including the case of the field proportional to $\cos ^{2}(\theta)$ as in equation (39). Moreover, the Onsager theory was resolved by Lee [19] to determine the orientation distribution for the case of elongational flow, a potential with the same symmetry as the potential above. In the following we restrict our considerations again to hard spherocylinders, whose bulk phase diagram was studied in the previous section.

\subsection{Theoretical description}

First of all, density functional theory provides a natural framework for including external fields. If a reasonable approximation for the functional of the excess free energy is known, the contribution due to an external field is obtained by adding the term

$$
\mathcal{F}^{\text {ext }}[\rho]=\int \mathrm{d} \boldsymbol{x} \rho^{(1)}(\boldsymbol{x}) U_{\text {ext }}(\boldsymbol{x})
$$


to the bulk functional. For an Onsager orientation distribution function (13), the contribution to the free energy from the external potential reads

$$
\mathcal{F}^{\mathrm{ext}}(\alpha)=-N U_{0}\left(1+\frac{2}{\alpha^{2}}-\frac{2 \operatorname{coth}(\alpha)}{\alpha}\right)
$$

In this section we shall also make use of the Gaussian parametrization

$$
g(\vec{\omega})=\mathcal{N}^{-1} \exp \left[\alpha P_{2}\left(\vec{\omega} \cdot \vec{\omega}_{0}\right)\right]
$$

where $P_{2}(x)=\left(3 x^{2}-1\right) / 2$ is the second Legendre polynomial, $\mathcal{N}$ is present to guarantee correct normalization and $\alpha$ is the single variational parameter. While there is no big difference when comparing these two parametrizations in the Onsager limit of the bulk [16,55], this could be changed when including an external field. We will therefore check the stability of our results with respect to these two parametrizations of $g(\vec{\omega})$.

The practical problem, however, is that there is no approximation available which reproduces all the phases correctly. Our MWDA, for example, failed to get a correct stability of the solid phases. Therefore we shall use the MWDA only for the isotropic, nematic and smectic-A phases. For the crystalline phases, on the other hand, we use the more empirical cell theory $[9,10]$ which also works variationally: the excess free energy is a functional of the effective size $\bar{R}$, which is itself a functional of the orientation distribution $g(\vec{\omega})$, i.e.

$$
\mathcal{F}^{\mathrm{exc}}([\bar{R}[g]])=\mathcal{F}^{\mathrm{exc}}([g(\vec{\omega})])=\mathcal{F}^{\mathrm{exc}}(\alpha) .
$$

We add the external coupling to the total free energies obtained in references $[9,10]$ and thus get a simple description for the solid phases in the presence of an external field. With this 'mixed' approach we get a reasonable description for any possible phases.

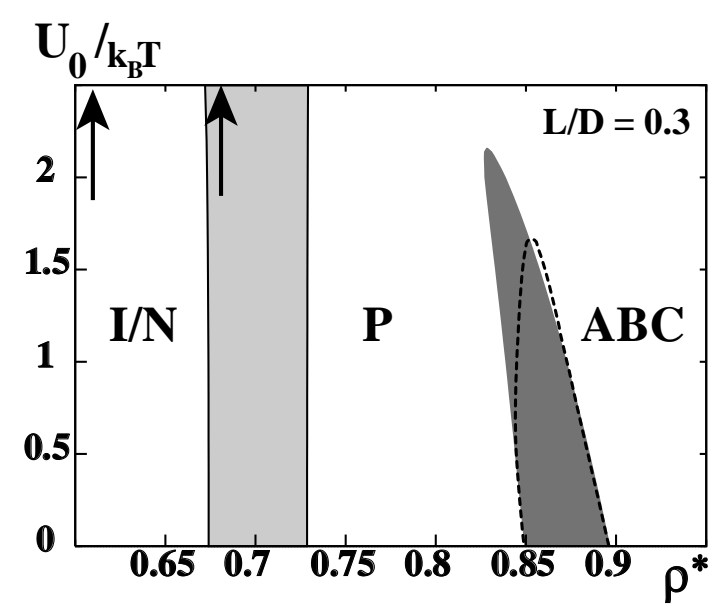

Figure 2. Hard spherocylinders with a length-to-width ratio of $p=0.3$ in an external field: the transition densities $\rho^{*}$ are plotted versus the strength of the external field $U_{0} / k_{\mathrm{B}} T$. Besides the nematic-crystal transition (light-shaded area) the critical behaviour of the plastic-ABC-solid transition is shown (dark-shaded area for an Onsager orientation distribution and broken curve for a Gaussian orientation distribution). The critical points are located at the top of the coexistence regions. The nematic-solid transition of strictly aligned HSC $U_{0} \rightarrow \infty$ is indicated by the two arrows on the upper axis. 


\subsection{Results for selected aspect ratios}

Results are obtained for three different aspect ratios $p=0.3,2,4$ for arbitrary densities and arbitrary field strengths. First we consider short rods $(p=0.3)$ with a plastic-fcc-solid transition. Then intermediate rods with $p=2$ are studied, showing no nematic phase in the absence of the external field. Finally for $p=4$ we consider the stability of all of the usual liquid-crystal phases except the crystal. We shall also discuss the phase behaviour versus the orientational order parameter $S=\left\langle P_{2}(\cos \theta)\right\rangle_{g(\vec{\omega})}$, which is easily evaluated in the case of the Onsager distribution function, $S=1+3 / \alpha^{2}-3 \operatorname{coth}(\alpha) / \alpha$.

3.3.1. Short rods: $p=0.3$. For short rods, the resulting phase behaviour versus reduced density and field strength is shown in figure 2 . In the presence of external fields, an isotropic fluid no longer exists, as the ordering effects turn the fluid into a nematic-like pre-ordered fluid. We use the term 'fluid' in the sense of this pre-ordered isotropic phase. The light-shaded area in figure 2 corresponds to the fluid/nematic-solid transition. The coexisting solid phase is a plastic crystal with an anisotropic orientational distribution induced by the field. The dark-shaded area corresponds to the plastic-ABC-solid coexistence regions: above a critical field strength of $U_{0}=2.16 k_{\mathrm{B}} T$ the plastic-ABC-crystal transition vanishes. As expected, the critical behaviour is mean-field-like, i.e. on approaching the critical point at $\rho_{\mathrm{c}}^{*}$, the coexistence densities behave as $\left|\rho^{*}-\rho_{\mathrm{c}}^{*}\right|^{1 / 2}$. Similarly one obtains for the orientational order parameter $\left|S-S_{\mathrm{c}}\right|^{1 / 2}$, where $S_{c}$ is the critical nematic order parameter. On the other hand, the fluid/nematic-solid transition remains first order. The limiting case of parallel hard spherocylinders [48], marked by the two arrows on the upper axis, is reached very slowly as $U_{0} \rightarrow \infty$.

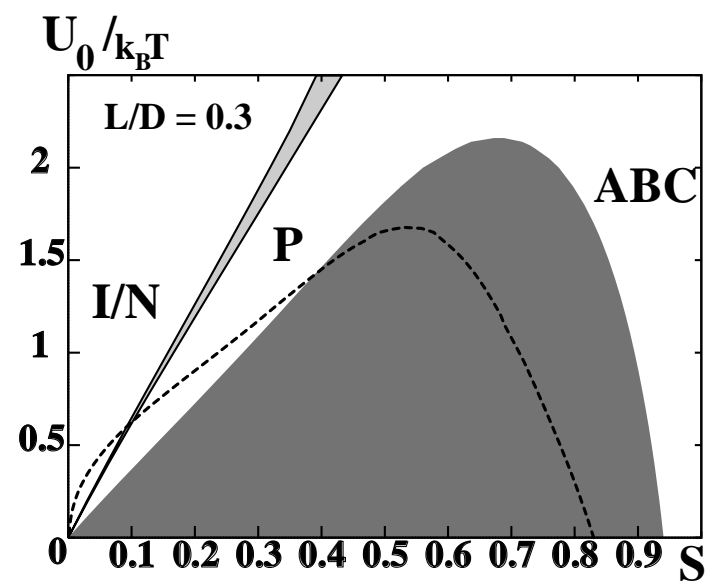

Figure 3. As figure 2, but now the strength of the external field $U_{0}$ is plotted versus the order parameter $S$ at the transition.

The transitions are also shown as a function of the orientational order parameter in figure 3 . The same topology of the phase diagram is observed.

Two other features are worth mentioning.

(a) First, the details of the transition in the absence of the field do not depend crucially on the chosen orientation distribution. This, however, is not true for the location of the critical point: replacing the orientation distribution of the Onsager type with a Gaussian 
orientation distribution (42) yields a critical point at $U_{0}=1.65 k_{\mathrm{B}} T$. The corresponding plastic-ABC-crystal transition is indicated as broken curves in figures 2 and 3.

(b) Second, by calculating the compressibility $\kappa=-(1 / V)(\partial V / \partial \mathcal{P})$ (where $\mathcal{P}$ is the pressure) the pre-ordered fluid/nematic phase has been checked for mechanical stability. As a result there was no transition from the pre-ordered isotropic phase into another nematic phase.

3.3.2. Medium rods: $p=2$. Contrary to the phase behaviour of short and long rods, the phase behaviour for medium rods is different for orientable particles and strictly aligned hard spherocylinders: whereas the former show for an aspect ratio of $p=2$ only an isotropic fluid and an $\mathrm{ABC}$-stacked solid (see figure 1), the latter exhibit besides the nematic phase a smectic-A and a solid ABC structure [48].

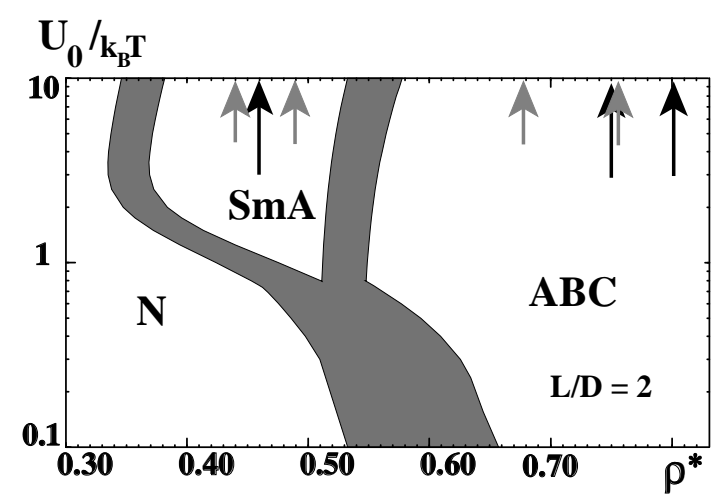

Figure 4. As figure 2, but now for spherocylinders with a length-to-width ratio of $p=2$. For small external fields, the HSC exhibit the typical phase behaviour of orientable rods, i.e. only an isotropic and a solid phase occur. For external fields larger than $U_{0}=0.83 k_{\mathrm{B}} T$, a smectic-A phase becomes stable. The nematic-smectic as well as the smectic-ABC transition in the limiting case of parallel hard spherocylinders are indicated by the black arrows (simulational results of reference [48]) and by the grey arrows (the approximative cell model). Note the logarithmic scale for the field strength.

The external field (39) continuously switches between these two limiting cases (see figure 4). Starting from an already orientationally ordered isotropic phase for a small nonvanishing external field, we first observe a nematic-ABC-solid transition. Beyond an external field of $U_{0}=0.83 k_{\mathrm{B}} T$ a smectic phase becomes stable. The cell theory free energy contains the case of strictly aligned hard spherocylinders. This limit is reached, however, only for very high field strengths. The coexisting densities are considerably smaller for finite field strengths than that for the strictly aligned case. This is also indicated in figure 4 , where the continuous nematic-smectic transition is shown by a black arrow and the results of the cell theory by the grey arrows on the upper axis. Additionally we compare the first-order smectic-solid transition which occurs at higher densities. Again the black arrow indicates the exact result while the cell theory predictions are shown by the grey arrow. The Onsager parametrization was used throughout our calculations in this case.

3.3.3. Long rods: $p=4$. In the absence of an external field, the isotropic-nematic transition is the first to occur upon increasing the density. On switching on the external field (39), this transition (the dark-shaded area in figure 5) shows a critical point at $U_{0}=0.055 k_{\mathrm{B}} T$. The behaviour is similar to that in the Onsager theory [17]. 


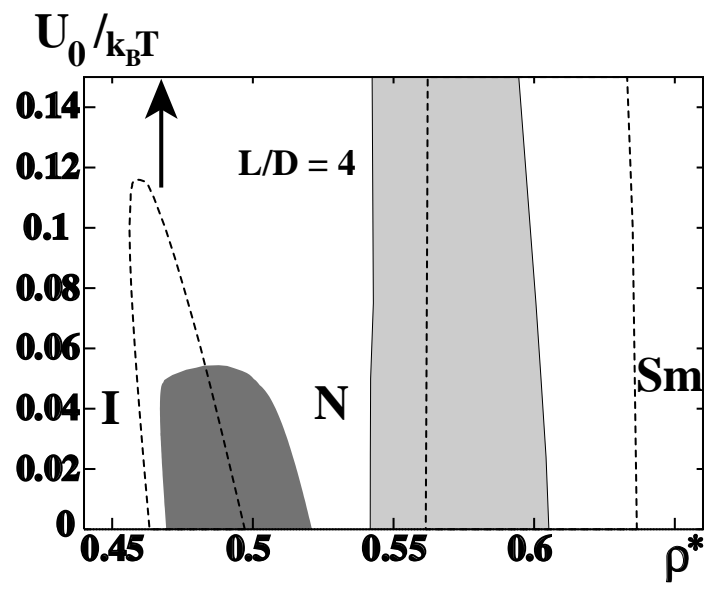

Figure 5. As figure 2, but now for spherocylinders with a length-to-width ratio of $p=4$. The isotropic-nematic transition (dark-shaded area) terminates in a critical point, whereas the nematicsmectic transition remains first order (light-shaded area). Again we include the results for the Gaussian orientation distribution as broken curves. The continuous transition from the nematic to the smectic phase in the case of parallel hard spherocylinders is indicated by the arrow on the upper axis.

Again it is worth mentioning that the details crucially depend on the chosen orientation distribution. For short HSC, the coexistence densities of the fluid/nematic-solid transition depend only slightly on the chosen orientation distribution. This is not the case for the isotropicnematic transition: the results for a Gaussian orientation distribution are included in figure 5 as broken lines. They result in a considerably higher critical field of $U_{0}=0.118 k_{\mathrm{B}} T$.

The transition from the nematic phase to the smectic phase is only slightly affected by the external field. The limiting case of parallel hard spherocylinders shows no measurable density jump at the nematic-smectic transition in simulations [48]. This limit, as indicated by the arrow on the upper axis in figure 5, is approached very slowly. Again the result is sensitive to the choice of the parametrization of the orientational distribution function. Finally,

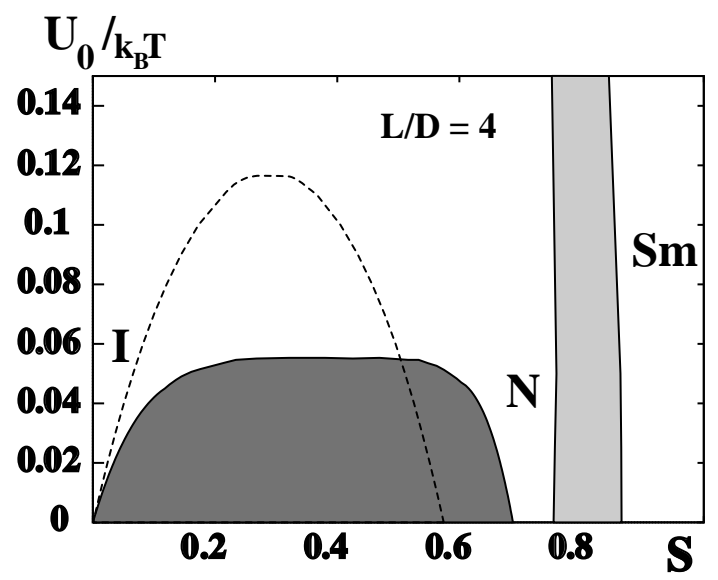

Figure 6. As figure 3, but now for hard spherocylinders with a length-to-width ratio of $p=4$. 
for comparison, the phase behaviour is shown as a function of the external field and the order parameter $S$ in figure 6.

\section{Conclusions}

To summarize: first, we have made an attempt to describe the full phase diagram of hard spherocylinders using the unifying framework of density functional theory-within our MWDA treatment, however, the crystalline phases were unstable with respect to the smectic phase; second, we have studied the influence of an external field coupling to the orientational degrees of freedom. The external field can both destroy and generate phase transitions. Phases which possess the same symmetry as far as the translational degrees of freedom are concerned, merge continuously into each other above a critical field strength. In particular, this was demonstrated for the isotropic-nematic and for the plastic-ABC-crystal transition. On the other hand, the field can stabilize phases which are unstable in the field-free case. This was demonstrated for the smectic phase. We have also shown that huge field strengths are required to achieve the asymptotic fully aligned limit. All of these theoretical predictions can in principle be verified by computer simulation or by experiments.

We finish with two remarks.

First, we comment on the factorization ansatz that we used in separating the orientational and translational degrees of freedom in the density parametrization. As is known from computer simulations, the factorization ansatz is well justified, in general. In only a small region of the smectic phase, a transverse interlayer order affecting up to one per cent of the particles is found in computer simulations [37,51], a small effect in a small range of the phase diagram, which we missed in our ansatz.

Second, more fundamentally, there is a real need to develop and apply density functional approximations which incorporate the correct packing constraints for hard bodies, such as the Rosenfeld fundamental measure functional [38-40]. The external field can be used formally to cross over from the orientable case to the fully aligned case and to achieve self-consistency; this is similar to the dimensional crossover discussed recently for hard spheres [40]. This problem is a great challenge for future studies.

\section{Acknowledgments}

We thank A M Denton and M Schmidt for helpful remarks. Financial support from the DFG (Gerhard-Hess-Programm) is gratefully acknowledged.

\section{Appendix. Expansion in spherical invariants}

For the expansion of the weight function in spherical harmonics we anticipate the conventions of Gray and Gubbins [11]. The weight function $w\left(\vec{r}_{12}, \vec{\omega}_{1}, \vec{\omega}_{2}\right)$ depends on the orientations of the two particles described by unit vectors $\vec{\omega}_{1}$ and $\vec{\omega}_{2}$ pointing along their axes and on the distance vector between the centres of mass $\vec{r}_{12}$ with the corresponding unit vector $\vec{\omega}_{12}$ : $\vec{r}_{12}=r_{12} \vec{\omega}_{12}$.

The quantity

$\Phi_{\Lambda}\left(\overrightarrow{\omega_{12}}, \vec{\omega}_{1}, \vec{\omega}_{2}\right)=\sum_{m_{1}=-l_{1}}^{l_{1}} \sum_{m_{2}=-l_{2}}^{l_{2}} \sum_{m=-l}^{l} C\left(l_{1} l_{2} l, m_{1} m_{2} m\right) \mathcal{Y}_{l_{1} m_{1}}\left(\vec{\omega}_{1}\right) \mathcal{Y}_{l_{2} m_{2}}\left(\vec{\omega}_{2}\right) \mathcal{Y}_{l m}^{*}\left(\overrightarrow{\omega_{12}}\right)$

where the $\mathcal{Y}_{l m}$ are spherical harmonics and the $C\left(l_{1} l_{2} l, m_{1} m_{2} m\right)$ are the Clebsch-Gordan coefficients is invariant under simultaneous rotations of $\vec{\omega}_{1}, \vec{\omega}_{2}$ and $\overrightarrow{\omega_{12}}$. 
Thus the weight function will be expanded as

$$
w\left(\vec{r}_{12}, \vec{\omega}_{1}, \vec{\omega}_{2}\right)=\sum_{\Lambda} \hat{w}_{\Lambda}\left(r_{12}\right) \Phi_{\Lambda}\left(\overrightarrow{\omega_{12}}, \vec{\omega}_{1}, \vec{\omega}_{2}\right)
$$

where the summation is running over the triple $\Lambda=\left(l_{1}, l_{2}, l\right) \in \mathbb{N}_{0}^{3}$.

The expansion coefficients read

$\hat{w}_{\Lambda}\left(r_{12}\right)=\frac{4 \pi}{2 l+1} \int_{S_{2}} \mathrm{~d}^{2} \vec{\omega}_{1} \int_{S_{2}} \mathrm{~d}^{2} \vec{\omega}_{2} w\left(r_{12} ; \vec{\omega}_{1}, \overrightarrow{\omega_{2}}, \overrightarrow{\omega_{12}}\right) \Phi_{\Lambda}^{*}\left(\overrightarrow{\omega_{12}}, \vec{\omega}_{1}, \vec{\omega}_{2}\right)$.

As they are rotationally invariant, we fix $\vec{\omega}_{12}$ pointing along the $z$-axis, $\vec{\omega}_{12}=\hat{e}_{z}$, to calculate them. The expansion coefficients thus reads with $\bar{m}=-m$

$$
\begin{aligned}
& \hat{w}_{\Lambda}\left(r_{12}\right)=\sqrt{\frac{4 \pi}{2 l+1}} \int \mathrm{d}^{2} \vec{\omega}_{1} \int \mathrm{d}^{2} \vec{\omega}_{2} \sum_{m=-\min \left(l_{1}, l 2\right)}^{\min \left(l_{1}, l_{2}\right)} C\left(l_{1} l_{2} l ; m \bar{m}, 0\right) . \\
& \times \mathcal{Y}_{l_{1} m}^{*}\left(\vec{\omega}_{1}\right) \mathcal{Y}_{l_{2} \bar{m}}^{*}\left(\vec{\omega}_{2}\right) w\left(r_{12}, \vec{\omega}_{1}, \vec{\omega}_{2} ; \vec{\omega}_{12}=\hat{e}_{z}\right) .
\end{aligned}
$$

Using the invariance of $w\left(\vec{r}_{12}, \vec{\omega}_{1}, \vec{\omega}_{2}\right)$ if one of the vectors $\vec{\omega}$ changes sign, only those expansion coefficients with even $l_{1}, l_{2}, l$ are non-zero.

\section{References}

[1] Barker J A and Henderson D 1976 What is 'liquid'? Understanding the states of matter Rev. Mod. Phys. 48587

[2] Bawden F C, Pirie N W, Bernal J D and Fankuchen I 1936 Liquid crystalline substances from virus-infected plants Nature 1381051

[3] Bolhuis P and Frenkel D 1997 Tracing the phase boundaries of hard spherocylinders J. Chem. Phys. 106666

[4] Curtin W A and Ashcroft N W 1985 Weighted-density-functional theory of inhomogeneous liquids and the freezing transition Phys. Rev. A 322909

[5] Denton A R and Ashcroft N W 1989 Modified weighted-density functional theory of nonuniform classical liquids Phys. Rev. A 394701

[6] Esposito M and Evans G T 1994 Isotropic, nematic and smectic A phases in fluids of hard spherocylinders Mol. Phys. 83835

[7] Evans G T 1992 Liquid crystal smectic-A phases and issues of geometry Mol. Phys. 761359

[8] Evans R 1992 Density functionals in the theory of nonuniform fluids Fundamentals of Inhomogeneous Fluids ed D Henderson (New York: Wiley) ch 3, p 85

[9] Graf H and Löwen H 1998 Phase diagram of tobacco mosaic virus solutions Phys. Rev. E submitted

[10] Graf H, Löwen H and Schmidt M 1997 Cell theory for the phase diagram of hard spherocylinders Prog. Colloid Polym. Sci. 104177

[11] Gray C G and Gubbins K E 1984 Fundamentals (Theory of Molecular Fluids vol 1) (Oxford: Clarendon)

[12] Hansen J-P and McDonald I R 1986 Theory of Simple Liquids (New York: Academic)

[13] Hanus J 1969 Effect of the molecular interaction between anisotropic molecules on the Kerr effect. Field-induced phase transition Phys. Rev. 178420

[14] Holyst R and Poniewierski A 1989 Nematic-smectic-A transition for perfectly aligned hard spherocylinders: application of the smoothed-density approximation Phys. Rev. Lett. 392742

[15] Hornreich R M 1985 Landau theory of the isotropic-nematic critical point Phys. Lett. A 109232

[16] Kayser R F and Raveche H J 1978 Phys. Rev. A 172067

[17] Khoklov A R and Semenov A N 1982 Influence of external field on the liquid-crystalline ordering in solutions of stiff-chain macromolecules Macromolecules 151272

[18] Lasher G 1970 Nematic ordering of hard rods derived from a scaled particle treatment J. Chem. Phys. 534141

[19] Lee S-D 1987 Comment on effects of elongational flow on the isotropic-nematic phase transition in rodlike systems J. Chem. Phys. 866567

[20] Lee S-D 1987 A numerical investigation of nematic ordering based on a simple hard-rod model J. Chem. Phys. 874972

[21] Lelidis I, Nobili M and Durand G 1993 Electric-field-induced change of the order parameter in a nematic liquid crystal Phys. Rev. E 483818

[22] Löwen H 1994 Melting, freezing and colloidal suspensions Phys. Rep. 237249 
[23] Marko J F 1988 Accurate calculation of isotropic-plastic and isotropic-nematic transitions in hard-ellipsoid fluid Phys. Rev. Lett. 60325

[24] Martin C, Kramer H, Johner C, Weyerich B, Biegel J, Deike R, Hagenbüchle M and Weber R 1995 Electric and magnetic field studies on rodlike fd-virus suspensions Macromolecules 283175

[25] McCoy J D, Singer S J and Chandler D 1987 A density functional treatment of the hard dumb-bell freezing transition J. Chem. Phys. 874853

[26] Mulder B 1987 Density-functional approach to smectic order in an aligned hard-rod fluid Phys. Rev. A 353095

[27] Nicastro A J and Keyes P H 1984 Electric-field-induced critical phenomena at the nematic-isotropic transition and the nematic-isotropic critical point Phys. Rev. A 303156

[28] Onsager L 1949 The effect of shape on the interaction of colloidal particles Ann. NY Acad. Sci. 51627

[29] Oxtoby D W 1991 Crystallization of liquids: a density functional approach Liquids, Freezing and Glass Transition (Les Houches Lectures vol LI) ed J-P Hansen, D Levesque and J Zinn-Justin (Amsterdam: Elsevier Science) p 147

[30] Parsons J D 1979 Nematic ordering in a system of rods Phys. Rev. A 191225

[31] Polson J M and Frenkel D 1997 First-order nematic-smectic transition for hard spherocylinders in the limit of infinite aspect ratio Phys. Rev. E 56 R6260

[32] Poniewierski A 1992 Nematic to smectic-A transition in the asymptotic limit of very long hard spherocylinders Phys. Rev. A 455605

[33] Poniewierski A and Holyst R 1988 Density-functional theory for nematic and smectic-A ordering of hard spherocylinders Phys. Rev. Lett. 612461

[34] Poniewierski A and Holyst R 1990 Density-functional theory for systems of hard rods Phys. Rev. A 416871

[35] Poniewierski A and Sluckin T J 1991 Phase diagram for a system of hard spherocylinders Phys. Rev. A 436837

[36] Press W H, Teukolsky S A, Vetterling W T and Flannery B P 1992 Numerical Recipes in C (Cambridge: Cambridge University Press)

[37] van Roij R, Bolhuis P, Mulder B and Frenkel D 1995 Transverse interlayer order in lyotropic smectic liquid crystals Phys. Rev. E 52 R 1277

[38] Rosenfeld Y 1989 Free-energy model for the inhomogeneous hard-sphere fluid mixture and density-functional theory of freezing Phys. Rev. Lett. 63980

[39] Rosenfeld Y 1990 Free-energy for the inhomogeneous hard-sphere fluid in $D$ dimensions: structure factors for the hard disc $(d=2)$ mixtures in simple explicit form Phys. Rev. A 425978

[40] Rosenfeld Y, Schmidt M, Löwen H and Tarazona P 1996 Fundamental-measure free energy density functional for hard spheres: dimensional crossover and freezing Phys. Rev. E 554245

[41] Singer S J and Mumaugh R 1990 Monte Carlo study of fluid-plastic crystal coexistence in hard dumb-bells J. Chem. Phys. 931278

[42] Singh U P and Mohanty U 1988 Molecular theory for freezing transition of hard ellipsoid and hard dumb-bell molecules Phys. Rev. A 384377

[43] Singh U P and Singh Y 1986 Molecular theory for freezing of a system of hard ellipsoids: properties of isotropic-plastic and isotropic-nematic transitions Phys. Rev. A $\mathbf{3 3} 2725$

[44] Smithline S J, Rick S W and Haymet A 1988 Density functional theory of freezing for molecular liquids J. Chem. Phys. 882004

[45] Somoza A M and Tarazona P 1988 Nematic-smectic-A-smectic-C transitions in systems of parallel hard molecules Phys. Rev. Lett. 612566

[46] Somoza A M and Tarazona P 1989 Density functional approximation for hard-body liquid crystals J. Chem. Phys. 91517

[47] Somoza A M and Tarazona P 1990 Nematic and smectic liquid crystals of hard spherocylinders Phys. Rev. A 41 965

[48] Stroobants A, Lekkerkerker H N W and Frenkel D 1986 Evidence for smectic order in a fluid of hard parallel spherocylinders Phys. Rev. Lett. 571452

[49] Tang J and Fraden S 1993 Magnetic-field-induced isotropic-nematic phase transition in a colloidal suspension Phys. Rev. Lett. 713509

[50] Tarazona P 1984 A density functional theory of melting Mol. Phys. 5281

[51] van Duijneveldt J and Allen M P 1997 Free energy barriers for interlayer diffusion in the smectic-A phase of hard spherocylinders Mol. Phys. 90243

[52] Varga S, Jackson G and Szalai I 1998 External field induced paranematic-nematic phase transitions of rod-like systems Mol. Phys. 93377

[53] Vega C and Monson P A 1997 Plastic crystal phases of hard dumb-bells and hard spherocylinders J. Chem. Phys. 1072696

[54] Vega C, Paras E and Monson P A 1992 On the stability of the plastic crystal phase of hard dumb-bell solids 
J. Chem. Phys. 978543

[55] Vroege G J and Lekkerkerker H N W 1992 Phase transitions in lyotropic colloidal and polymer liquid crystals Rep. Prog. Phys. 551243

[56] Wojtowicz P J and Sheng P 1974 Critical point in the magnetic field-temperature phase diagram of nematic liquid crystals Phys. Lett. A $\mathbf{4 8} 235$

[57] Xu H, Lekkerkerker H N W and Baus M 1992 Nematic-smectic A and nematic-solid transitions of parallel hard spherocylinders from density functional theory Europhys. Lett. 17163 